\title{
Preliminary Radiation Analysis of the Total Ionizing Dose for the Resource Prospector Mission
}

\author{
Kristina Rojdev ${ }^{1}$ \\ NASA-JSC, Houston, TX, 77058 \\ Allan J. Tylka² \\ Retired NASA-GSFC, Greenbelt, MD, 20771 \\ and \\ William Atwell ${ }^{3}$ \\ Retired Boeing Research \& Technology, Houston, TX, 77059
}

\begin{abstract}
NASA's Resource Prospector (RP) is a collaborative project between multiple centers and institutions to search for volatiles at the polar regions of the Moon as a potential resource for oxygen and propellant production. The mission is rated Class $D$ and will be the first In-Situ Resource Utilization (ISRU) demonstration on the lunar surface and at the lunar poles. Given that this mission is rated Class $D$, the project is considering using commercial off the shelf (COTS) electronics parts to reduce cost. However, COTS parts can be more susceptible to space radiation than typical aerospace electronic parts and carry some additional risk. Thus, prior to parts selection, having a better understanding of the radiation environment can assist designers in the parts selection process. The focus of this paper is to provide a preliminary analysis of the radiation environment from launch, through landing on the surface, and some surface stay as an initial step in determining worst case mission doses to assist designers in screening out electronic parts that would not meet the potential dose levels experienced on this mission.
\end{abstract}

\section{Nomenclature}

$\begin{array}{ll}c G y & =\text { centigray } \\ C I R & =\text { co-rotating interaction region } \\ C O T S & =\text { commercial off the shelf } \\ G C R & =\text { Galactic cosmic ray } \\ G L E & =\text { Ground level event } \\ \text { LEO } & =\text { low Earth orbit } \\ H Z E T R N & =\text { high charge and energy transport } \\ I S R U & =\text { In-Situ Resource Utilization } \\ M e V & =\text { Megaelectron volt } \\ p f u & =\text { particle flux unit } \\ R P & =\text { Resource Prospector } \\ R P M & =\text { Resource Prospector Mission } \\ S P E & =\text { Solar Particle Event }\end{array}$

\section{Introduction}

7 HE Resource Prospector Mission (RPM) is planning to launch a lander, rover and science payload to the lunar poles. This mission is rated as Class $\mathrm{D}$ and thus, has the opportunity to more tightly manage and accept greater

${ }^{1}$ Aerospace Engineer, Systems Engineering and Test, 2101 NASA Parkway, MC EA351, and AIAA Member.

${ }^{2}$ Astrophysicist, Heliophysics Science Division, Code 672.

${ }^{3}$ Retired Boeing Technical Fellow, 16623 Park Green Way, and AIAA Associate Fellow. 
levels of risk. In following with this philosophy, the project is considering the use of Commercial off the Shelf (COTS) electronic parts for cost saving benefits. At the same time, the project needs to understand the radiation environment that these parts may experience and to which they may be susceptible so that the project can eliminate those parts that do not meet minimum qualifications.

The typical process of determining the total ionizing dose to electronic parts is to pick the worst case scenario solar particle event and estimate the total dose delivered to the part from that event. However, these worst case events occur infrequently and are more common during periods when the sun is undergoing intense activity. Additionally, the sun has recently entered a period of diminished activity and solar physicists are predicting that this diminished activity will occur for some time..$^{1-3}$ Therefore, using the typical process may lead to either eliminating parts that may be acceptable or increased mass and cost to shield some of these COTS parts.

Another approach would be to investigate the mission timeframe and the predicted sun spot number for that timeframe. Then select solar particle events (SPEs) more in-line with typical solar activity of the predicted sunspot number to more accurately estimate the total ionizing dose to electronic parts. ${ }^{4}$ This approach has been baselined as a preliminary step in estimating total ionizing dose to electronic parts for the RP lander, and will be discussed in detail below.

\section{Background}

\section{A. RP Mission Overview}

The Resource Prospector Mission (RPM) is a spacecraft with a rover and payload that will be landing on the lunar surface to investigate ice in the polar regions as a potential resource for oxygen and propellant production. At the time of this analysis, the mission was scheduled to launch in April 2018 and the following discussion will reflect that launch date. However, the launch date of the mission has been in flux and may not occur until 2020. The current nominal trajectory includes a small amount of time in Low Earth Orbit (LEO), a 5.5 day transfer from Earth orbit to Lunar orbit, and one day on the lunar surface performing data collection. Other trajectories to be considered are 60 day and 90 day transfers, as well as 7 day and 30 day lunar surface operations.

\section{B. Radiation Environments}

Given the nominal trajectory of the mission, there are three primary radiation sources to which the vehicle will be exposed. In LEO, the main source of radiation will be a result of the Van Allen Radiation belts surrounding the Earth. This radiation tends to be a constant radiation source when in LEO. Fortunately, the vehicle spends little time in LEO and will not incur a large dose as a result of the LEO environment. In transit and on the lunar surface, the primary sources of radiation to the spacecraft will be solar particle events (SPE) and galactic cosmic rays (GCR).

SPEs emanate from the sun and their frequency and intensity is in accordance with the solar cycle, as shown in Figure 1. The solar cycle is approximately eleven years in length and contains periods of high activity, known as "solar maximum," and periods of low activity, known as "solar minimum." During solar maximum, there tend to be more frequent and higher intensity SPEs, as is evident in Figure 1.

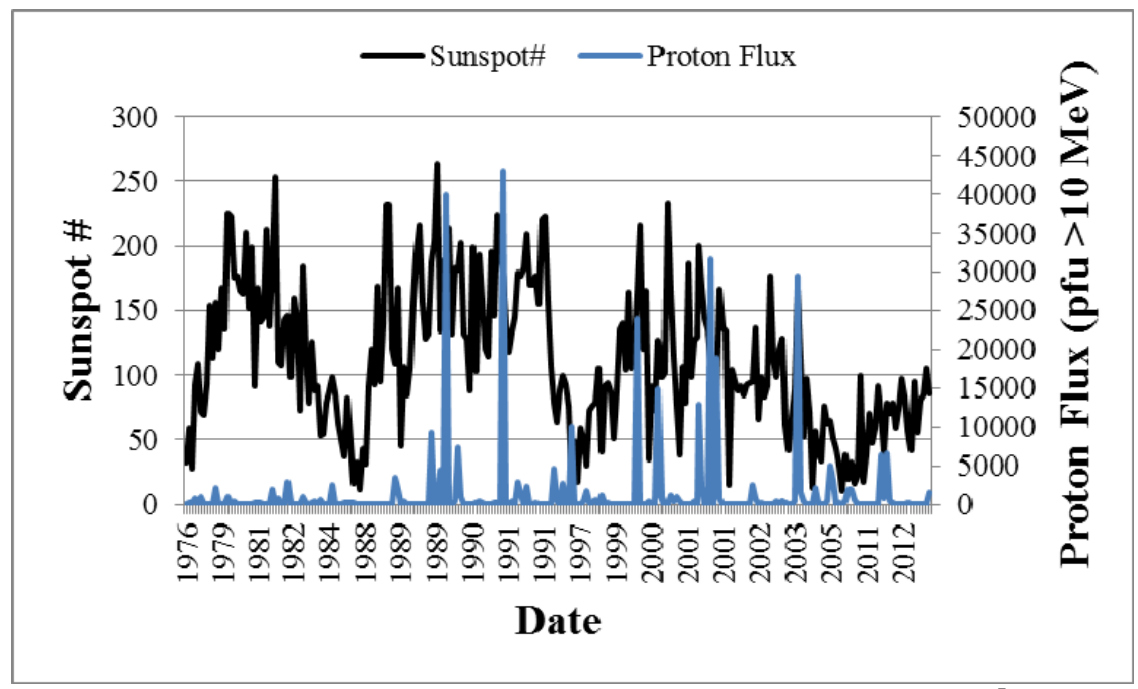

Figure 1: Solar cycle plotted with SPE intensity (data gathered from NOAA ${ }^{5}$ and SWPC ${ }^{6}$ ). 
GCRs originate outside the solar system and are very high energy particles, consisting of stripped nuclei ranging from hydrogen (proton) to iron. The GCR environment also varies with the solar cycle in an inverse relationship, such that when the sun is undergoing intense activity, the GCR is at a lower intensity (Figure 2).

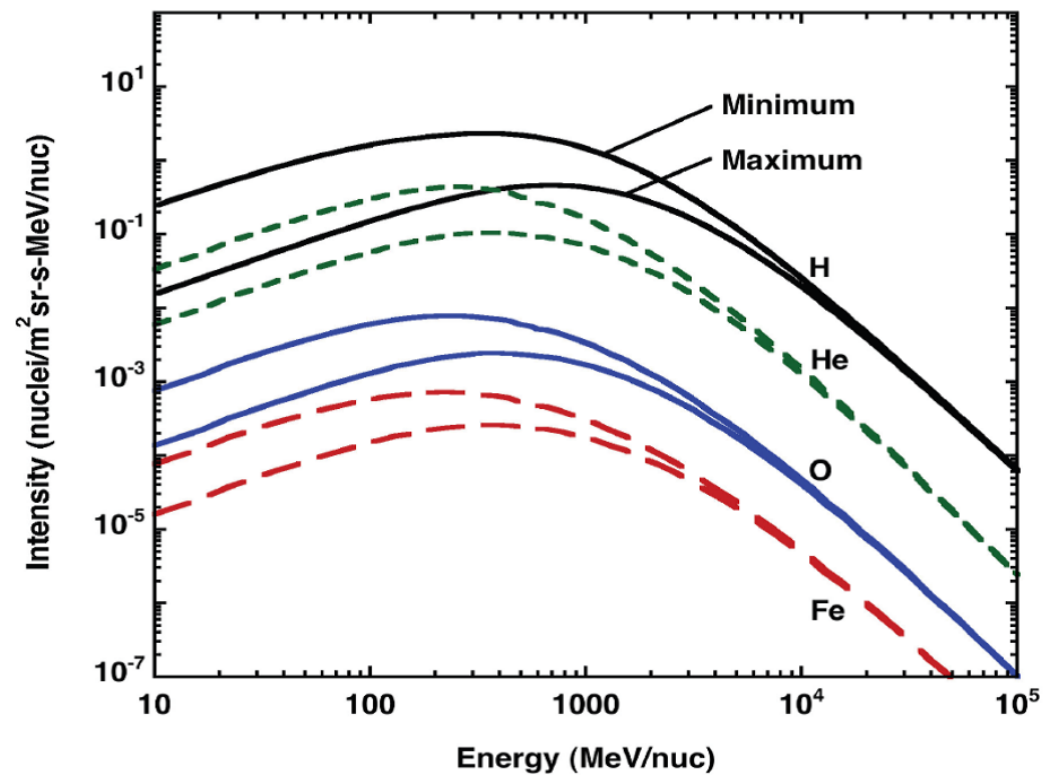

Figure 2: Differential fluence of several GCR elemental species at solar minimum and solar maximum conditions. ${ }^{7}$

\section{Methods}

\section{A. Solar Cycle Analysis and Environment Selection}

Given the launch date of April 2018, an analysis was undertaken of this date to better understand the radiation conditions that are predicted for this time period. The data from NOAA ${ }^{8}$ shows the following graph of the current solar cycle (Figure 3), and the predicted sunspot numbers for the future dates of the cycle.

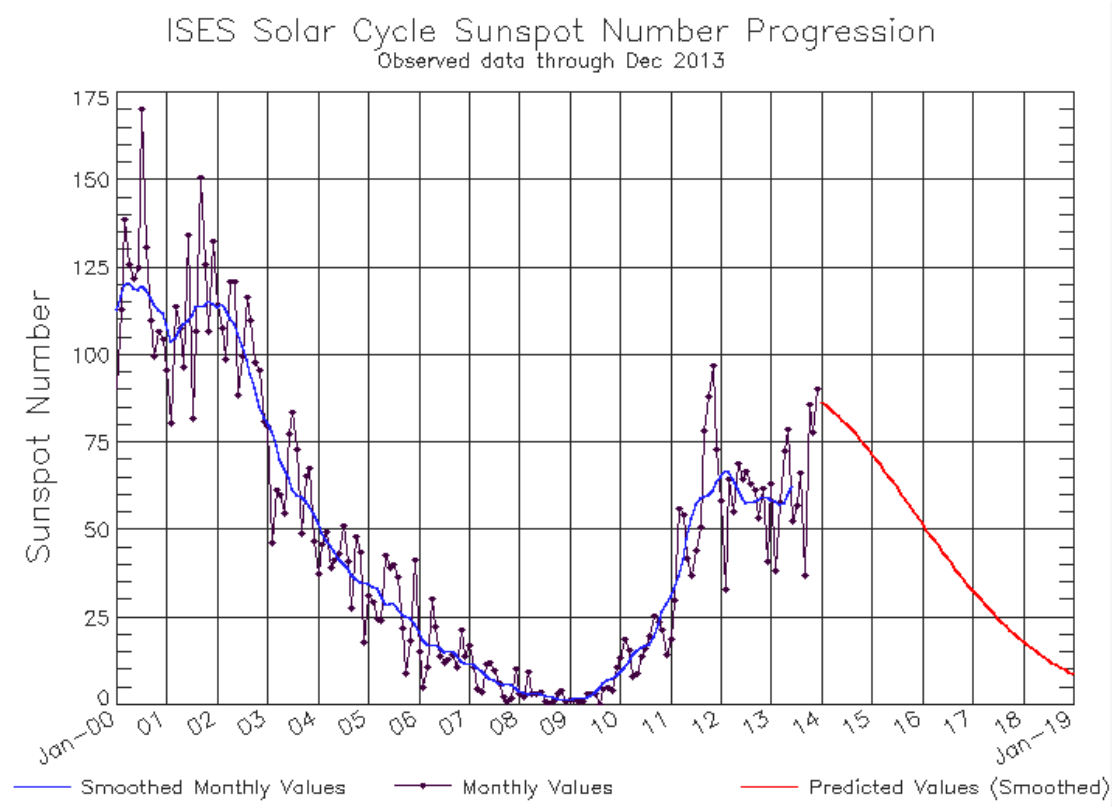

Updated 2014 Jan 6

Figure 3: Solar cycle 24 prediction ${ }^{8}$.

American Institute of Aeronautics and Astronautics 
The graph shows that 2018 will be a period in the solar cycle that is heading towards solar minimum. In solar minimum, there tends to be less frequent and lower intensity SPEs, whereas there is a higher flux of GCRs. The corresponding tabulated data ${ }^{9}$ to Figure 3 show that the predicted sunspot number for April 2018 is $15.4 \pm 10$.

Using this prediction and the data gathered in Figure 1, the areas of historical solar cycle relevance can be singled out (Figure 4). As can be seen from the graph, these areas have relatively few SPEs and are low intensity when compared with some of those SPEs that occured during solar maximum conditions.

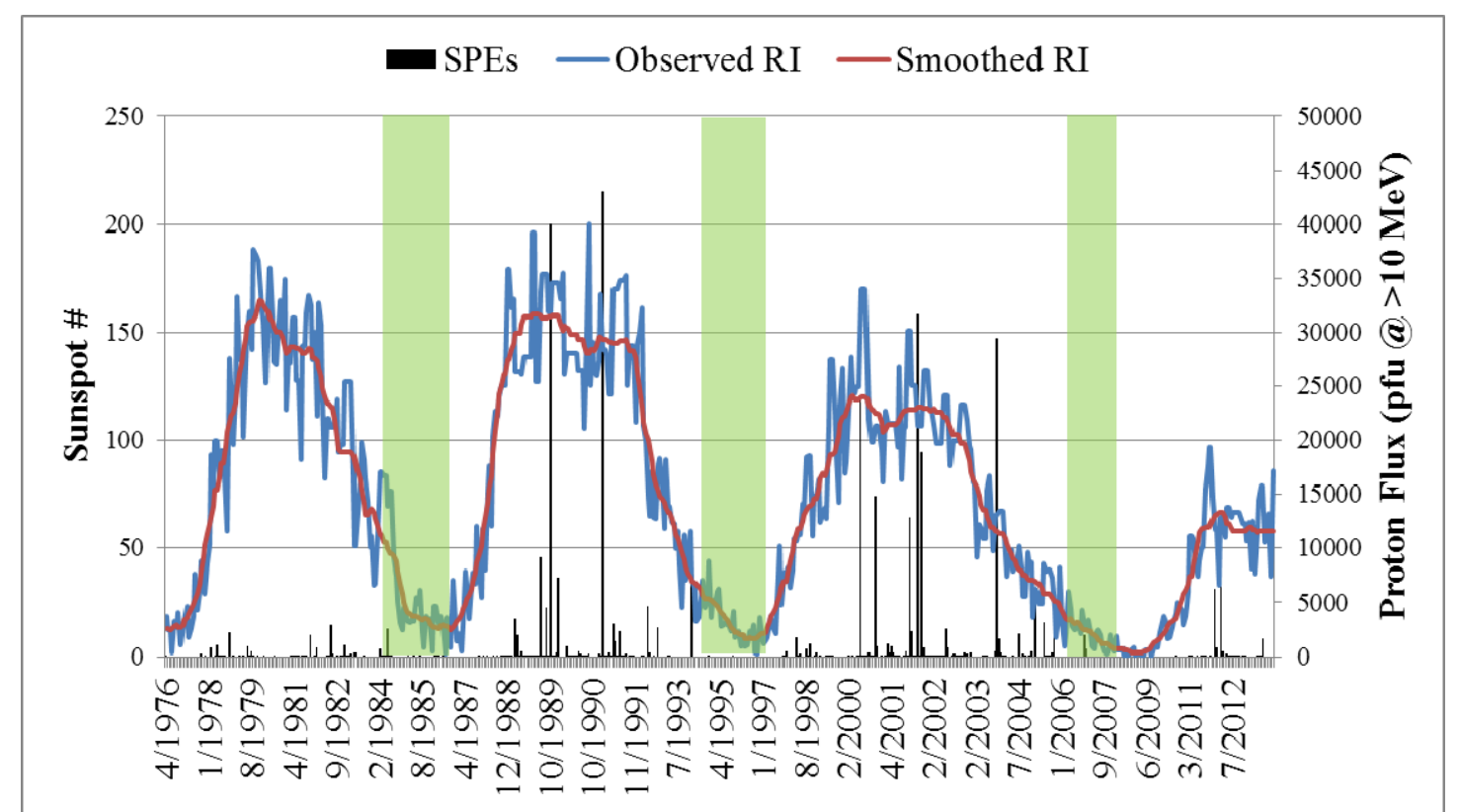

Figure 4: SPE intensity (proton flux) plotted with the sunspot numbers from 1976 to 2012. The areas highlighted in green represent the range of sunspot numbers that are in accordance with the predicted sunspot values for April 2018 and are heading into solar minimum.

Pulling out the data from these areas shows that there were approximately ten SPEs during this time period, which have been combined to show monthly values (Table 1). Of these events, December 2006 had very high proton fluxes and one of the events was a ground-level event (GLE). GLEs have very large energies (greater than $100 \mathrm{MeV}$ ) and can create secondary neutrons that are picked up by neutron monitoring stations all over the surface of the Earth. These tend to be rare events, but would be a worst case scenario for this type of mission.

Table 1: SPEs that occurred during the same range of sunspot number as the April 2018 prediction, and similar location in the solar cycle. ${ }^{10}$ These proton fluxes are peak fluxes for the SPEs during that month.

\begin{tabular}{ccc} 
Date & $\begin{array}{c}\text { Smoothed } \\
\text { Sunspot \# }\end{array}$ & $\begin{array}{c}\text { Peak Proton Flux } \\
\text { (pfu @ > 10 MeV) }\end{array}$ \\
\hline $\mathbf{1 / 1 9 8 5}$ & 20.5 & 14 \\
$\mathbf{4 / 1 9 8 5}$ & 18.3 & 160 \\
$\mathbf{7 / 1 9 8 5}$ & 17.4 & 140 \\
$\mathbf{2} / \mathbf{1 9 8 6}$ & 13.1 & 130 \\
$\mathbf{3 / 1 9 8 6}$ & 13 & 21 \\
$\mathbf{5 / 1 9 8 6}$ & 14.3 & 16 \\
$\mathbf{1 0 / 1 9 9 5}$ & 12.1 & 63 \\
$\mathbf{1 2 / 2 0 0 6}$ & 12.1 & 1980 \\
\hline
\end{tabular}

These SPEs were analyzed and the differential spectra are plotted below. Other than the December 2006 events, all the spectra above $100 \mathrm{MeV}$ are based on a power law extrapolation due to a lack of measurements at these higher 
energies. Therefore, the intensities above $100 \mathrm{MeV}$ should be treated as upper limits. The following section will provide more detail on the doses resulting from these SPEs.

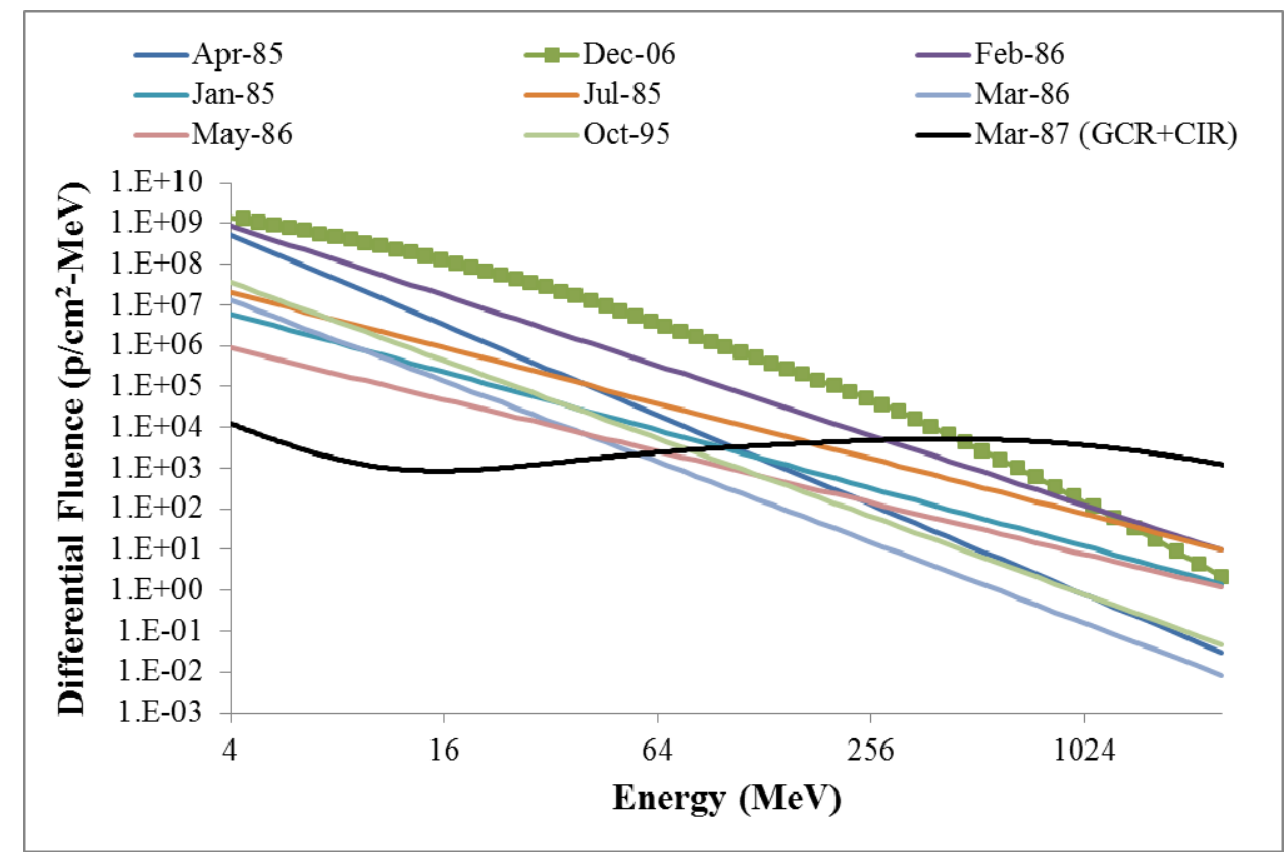

Figure 5: Differential Spectra of SPEs selected for analysis. The GCR differential spectrum for the month of March 1987 is also plotted in black for comparison. The co-rotating interaction regions (CIR) cause the rise in GCR proton intensities around $10 \mathrm{MeV}$ and below.

For the GCR environment, March 1987 was chosen for analysis because this environment occurred during solar minimum and March 1987 had a sunspot number of 14.7, similar to the April 2018 predicted sunspot number. The LEO environment is from April 1965, during solar minimum, which had a sunspot number of 6.8. These environments are pre-programmed into the transport code used for the analysis discussed in the next section.

\section{B. Radiation Dose Calculations}

To determine the total mission dose to the spacecraft, a one-dimensional, space-marching formulation of the Boltzmann transport equation with a straight-ahead approximation is used. This software is known as the highcharge-and-energy transport program $\left(\mathrm{HZETRN}^{11}\right)$ and is used by designers to provide quick and accurate dose information.

As mentioned in the previous section, both the GCR and LEO environments were already pre-programmed into HZETRN. However, the SPEs were not and the differential spectra (Figure 5) were input as user-defined environments to produce doses based on those environments.

For this analysis, no specific information was given on the thicknesses of the spacecraft. Thus, the assumptions were made that the vehicle was primarily aluminum and the shielding profile ranged from 0.1 to $250 \mathrm{~g} / \mathrm{cm}^{2}$ (0.04 to $92.59 \mathrm{~cm})$.

Given these assumptions of environments and shielding, the following dose data resulted from HZETRN for the LEO environment (Figure 6), GCR environment (Figure 6), and the various SPEs (Figure 7). Note that both LEO and GCR are a dose per day because they are constant background radiation sources, whereas the SPEs are a dose per event. 


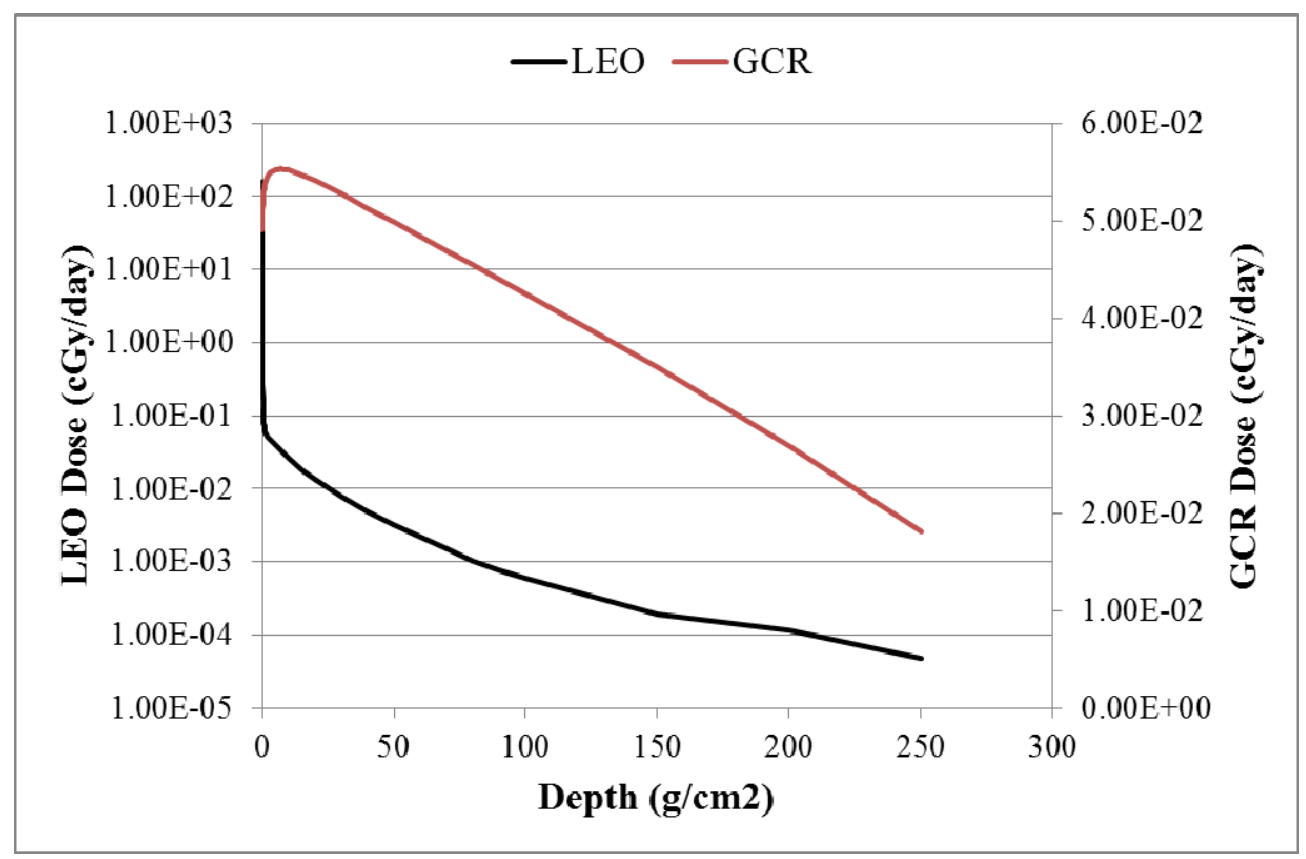

Figure 6: LEO (black) and GCR (maroon) Dose vs. Depth.

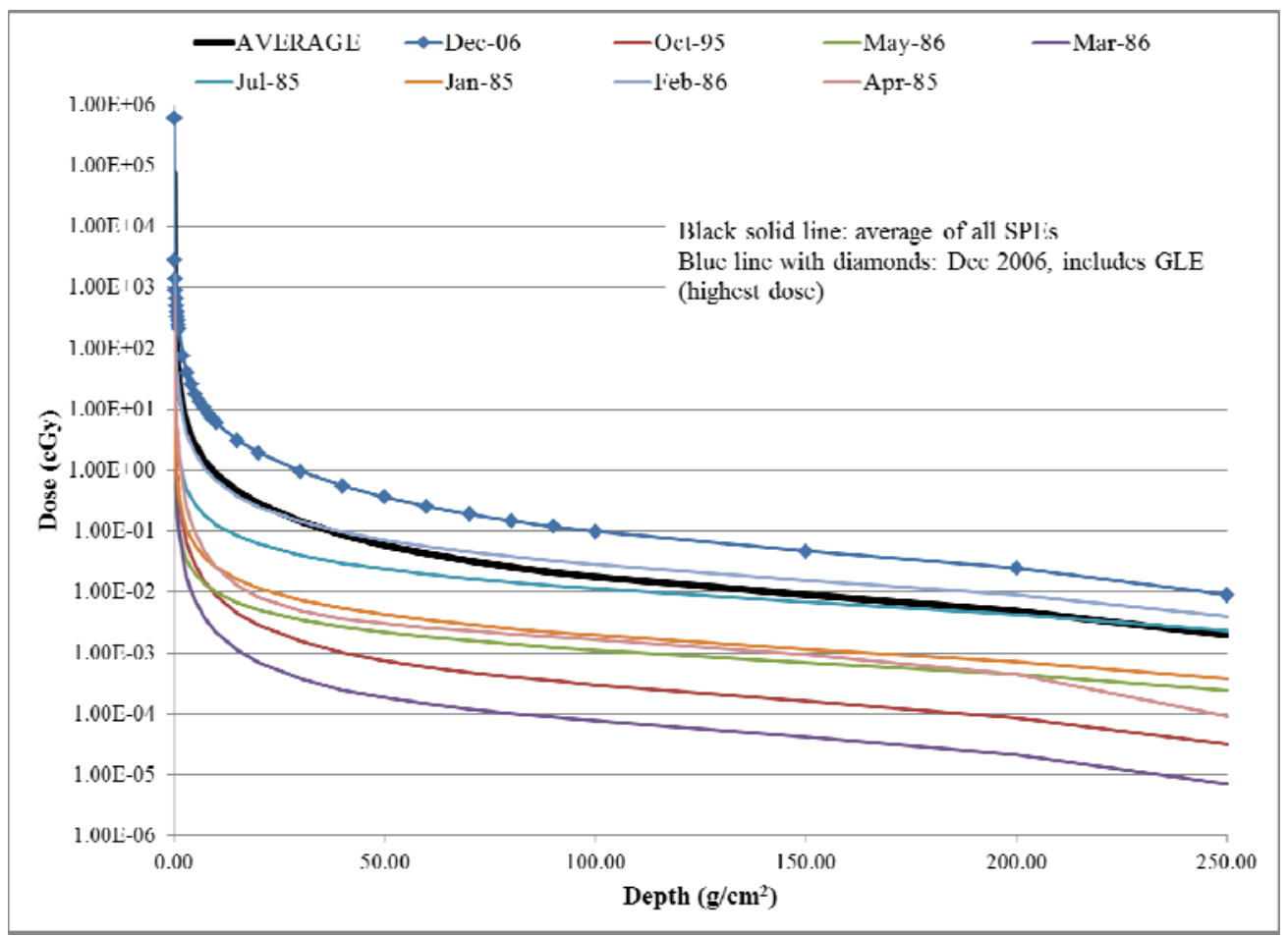

Figure 7: Dose vs. Depth for SPEs. The black line is an average of all the SPEs, and the blue line with diamonds is the SPEs from December 2006, which included the GLE.

\section{Mission Calculations}

For the different phases/parts of a mission, the dose is additive. Therefore, the dose resulting from exposure to the different environments was added in a logical fashion to provide the total mission dose. To determine the number of SPEs per mission case, the historical time periods of interest (Figure 4) were analyzed to determine worst case scenarios for SPE exposure (Figure 8). 
From the figure below, the worst case number of SPEs in one month is two, for two and three months is three, and for four months is four. These worst case numbers were used in the following calculations.

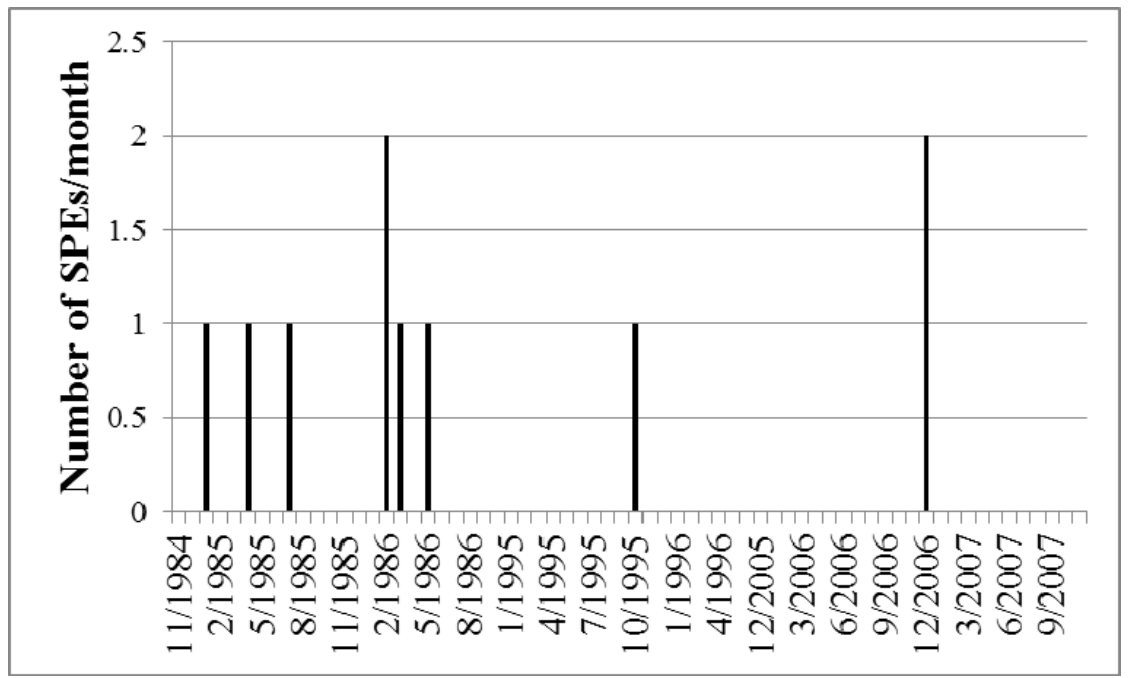

Figure 8: Number of SPEs per month for the three solar cycles heading towards solar minimum of historical significance.

In addition, the total dose delivered to the vehicle when on the lunar surface will be half of the exposure in deep space. This is due to the lunar volume shielding the vehicle on one side.

Using these worst case values for the SPEs and given the RP requirements, the following equations were used to perform the mission calculations.

\section{5.5 Day Transfer}

For the 5.5 day transfer case, the vehicle will spend a short amount of time in LEO (0.01 days) with 5.5 days in deep space (GCR and SPE exposure). The potential surface stay times are one day, seven days, and 30 days. Given that the total time for each of these potential missions is approximately or less than one month, the worst case SPE exposure will be two SPEs that occur during the transit period.

1 day on surface: $0.01 \times L E Q+2 \times S P E+5.5 \times G C R+\frac{1 \times G C R}{2}$

7 days on surface: $0.01 \times L E O+2 \times S P E+5.5 \times G C R+\frac{7 \times G G R}{2}$

30 days on surface: $0.01 \times L E Q+2 \times S P E+5.5 \times 6 C R+\frac{80 \times G C R}{2}$

\section{60 Day Transfer}

For the 60 day transfer case, the vehicle will spend the same amount of time in LEO ( 0.01 days) with 60 days in deep space (GCR and SPE exposure). The potential surface stay times are again one day, seven days, and 30 days. The total times for each of these potential missions are two or three months, therefore the worst case SPE exposure will be three SPEs during the transit phase.

1 day on surface: $0.01 \times L E Q+3 \times S P E+60 \times G C R+\frac{1 \times G G R}{2}$

7 days on surface: $0.01 \times L E Q+8 \times S P E+60 \times G C R+\frac{7 \times Q C A}{2}$

30 days on surface: $0.01 \times L E O+8 \times S P E+60 \times G C R+\frac{80 \times 9 C R}{2}$

\section{90 Day Transfer}

For the 90 day transfer, the vehicle will spend the same amount of time in LEO (0.01 days) with 90 days in deep space (GCR and SPE exposure). The potential surface stay times are the same as the previous two cases. The total times for these potential missions are three or four months. Therefore the worst case SPE exposure will be three 
SPEs for the one day and seven day surface stays, all occurring in transit, but four SPEs for the 30 day surface case where one SPE occurs while the vehicle is on the surface.

1 day on surface: $0.01 \times L E Q+8 \times S P E+90 \times G C R+\frac{1 \times Q C R}{2}$

7 days on surface: $0.01 \times L E Q+8 \times S P E+90 \times G C R+\frac{7 \times Q C R}{2}$

30 days on surface: $0.01 \times L E Q+8 \times S P E+900 \times G C R+\frac{80 \times G C R}{2}+\frac{1 \times S P E}{2}$

\section{Results and Discussion}

Using the averaged SPE dose (black line in Figure 7), the doses from Figure 6 for the LEO and GCR environments, and the above calculations, gives the following result for the total mission doses (Figure 9 and Figure 10).

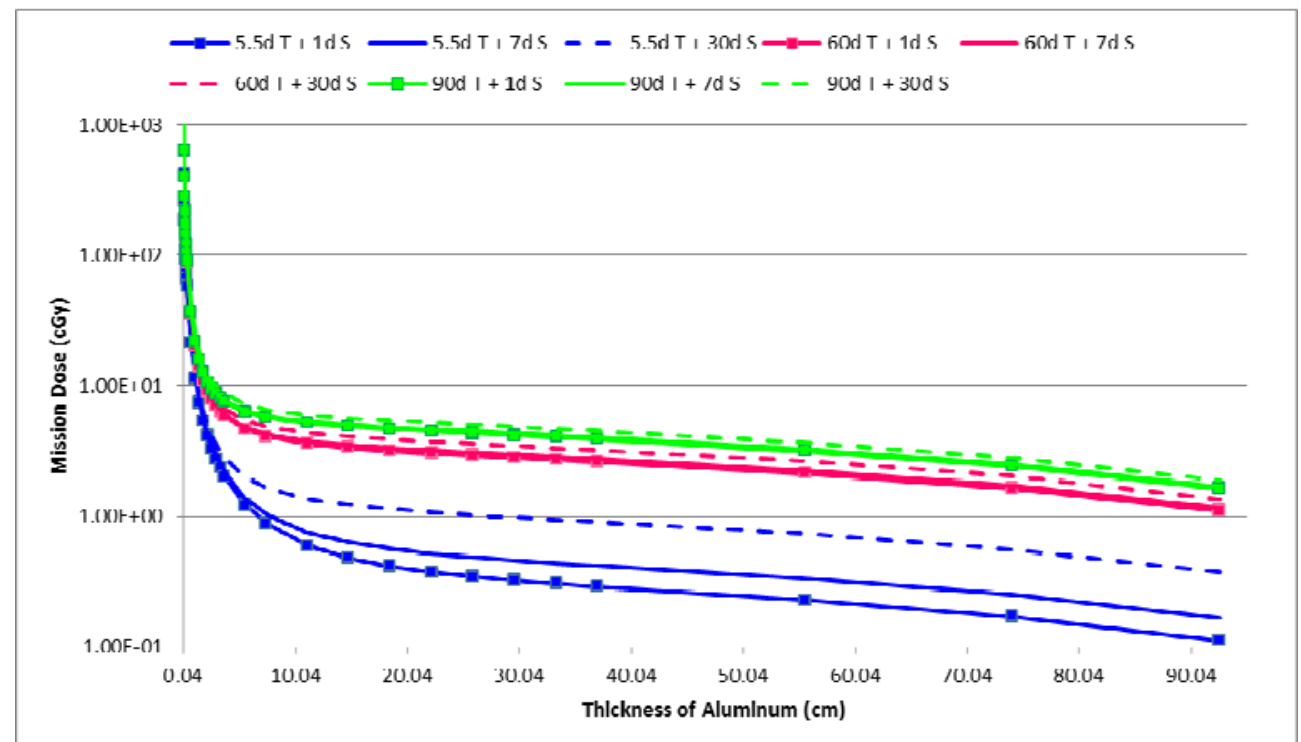

Figure 9: The total mission doses for each case with the averaged SPE environment. The thickness of aluminum is given in $\mathrm{cm}$ for this graph to make it more useful to designers. In the legend, $\mathrm{T}$ is the total time in transfer and $S$ is the total time on the lunar surface. 


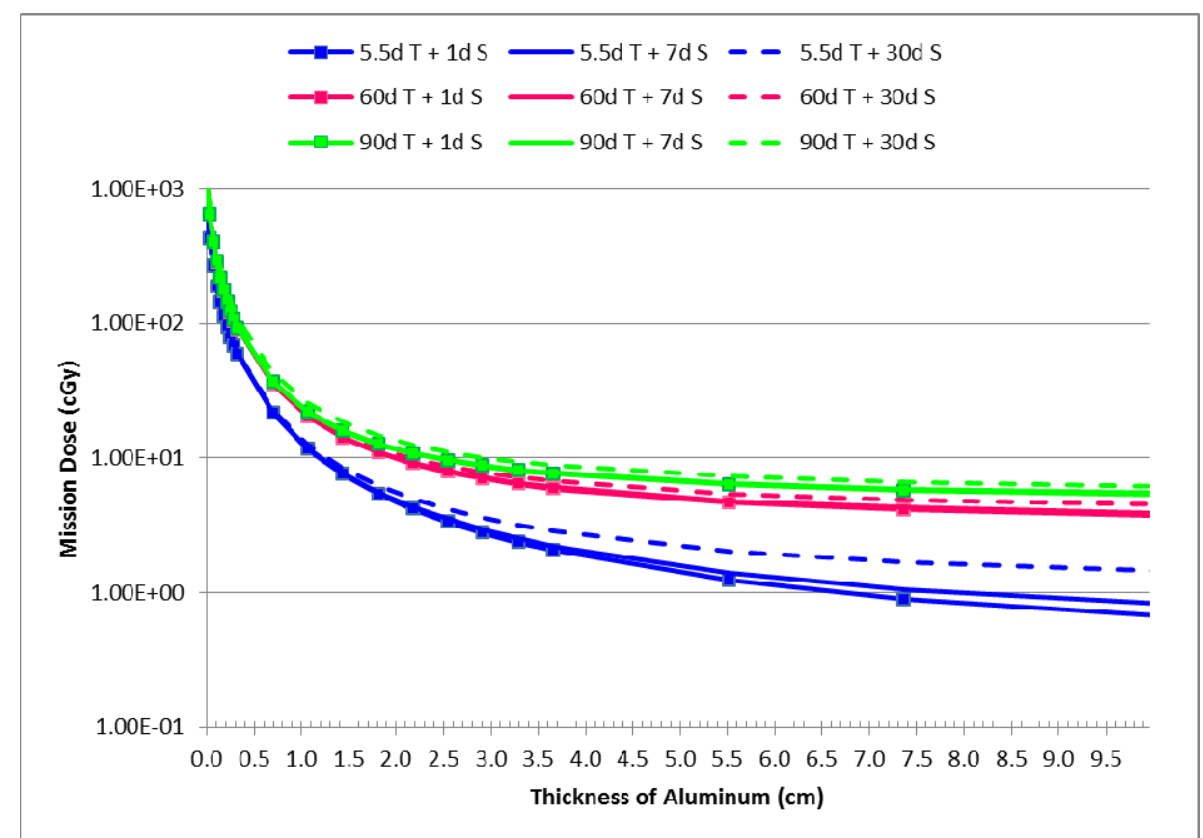

Figure 10: A zoomed in version of Figure 9 showing shielding thicknesses ranging from $0.04-10 \mathrm{~cm}$.

To provide the worst case dose for the mission timeframe, the combined SPEs in 12-2006 needs to be used instead of the average SPE because it had a GLE. Thus, the worst case total mission dose results are shown in Figure 11 and Figure 12.

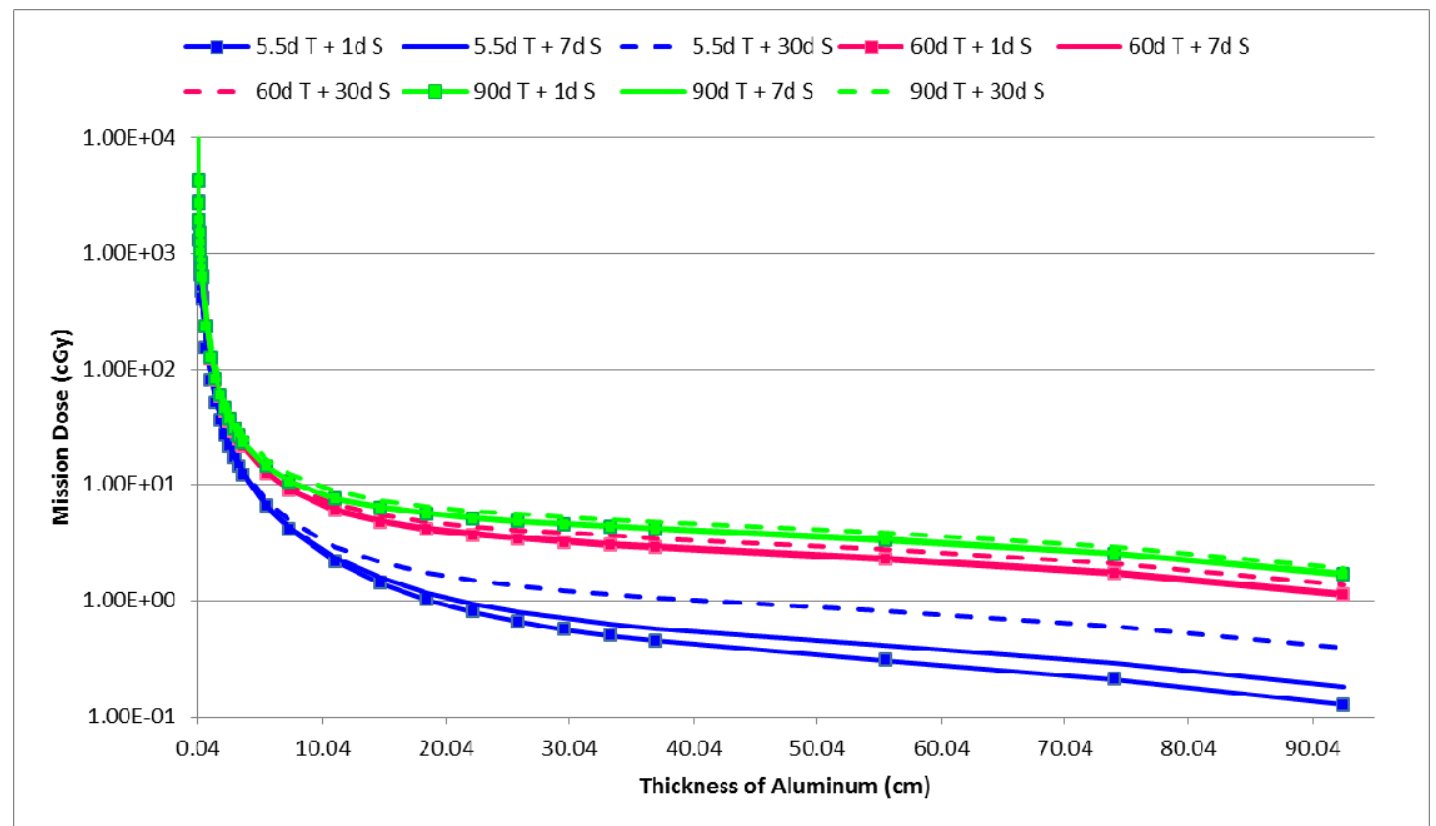

Figure 11: The worst case total mission doses for each scenario using 12-2006 as the SPE environment. The thickness of aluminum is given in $\mathrm{cm}$ for this graph to make it more useful to designers. In the legend, $\mathrm{T}$ is the total time in transfer and $S$ is the total time on the lunar surface. 


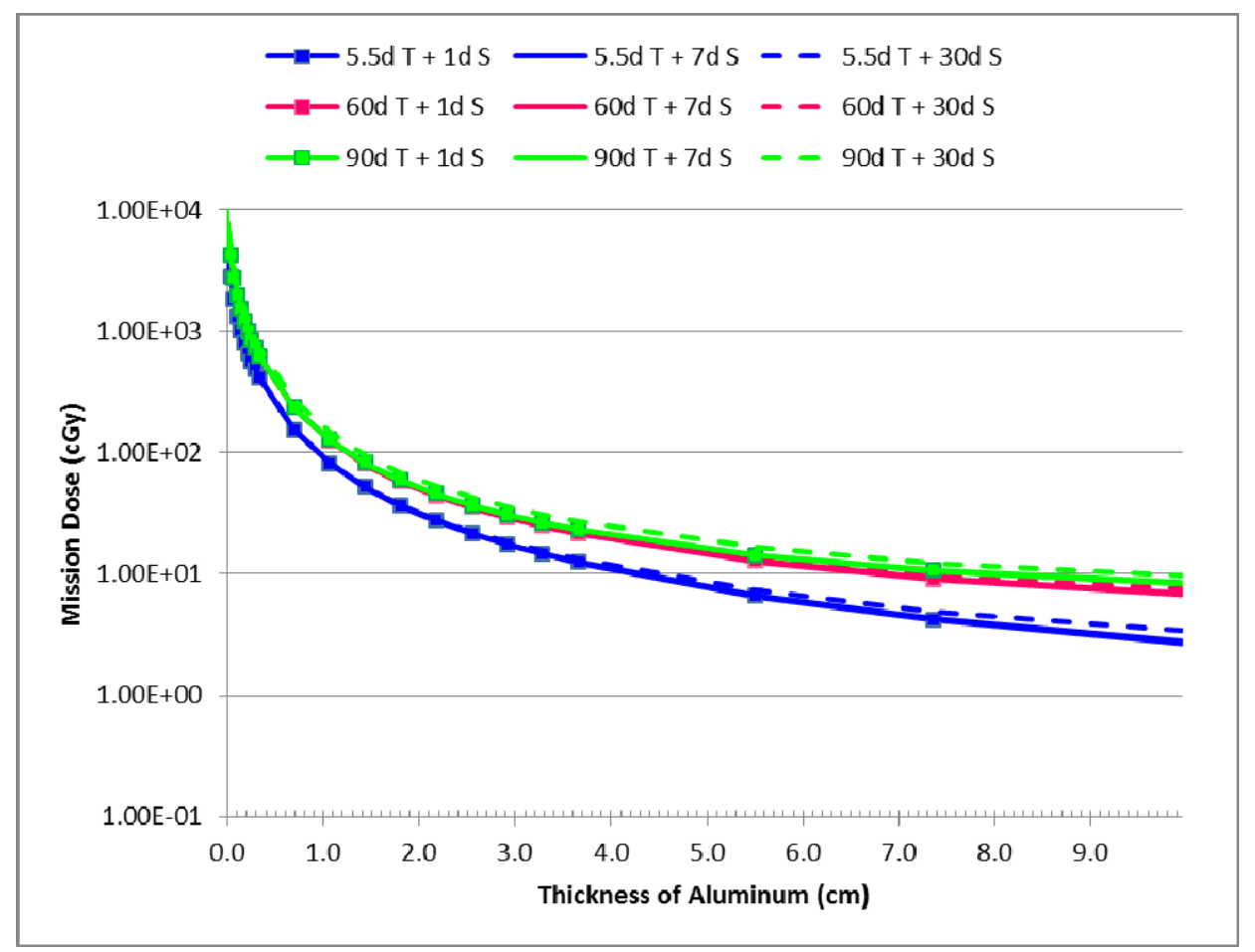

Figure 12: A zoomed in version of Figure 11 showing shielding thicknesses ranging from $0.04-10 \mathrm{~cm}$.

Figure 9 and Figure 11 are generally very similar. However, Figure 11 shows greater doses for thicknesses of aluminum that are less than $\sim 30 \mathrm{~cm}$ when considering missions with the 5.5 day transfer.

\section{Conclusion and Recommendations}

In general, the doses for any of these mission cases are pretty small. However, as preliminary steps in helping designers choose electronic components, it is recommended to use the total ionizing dose values from the worst case mission scenarios (Figure 11) to estimate the approximate dose to a part at a given thickness of shielding for the location of the part on the spacecraft.

\section{References}

${ }^{1}$ Livingston, W., and Penn, M., “Are Sunspots Different During This Solar Minimum?,” Eos Trans. AGU, 90(30), 257-258, 2009, doi:10.1029/2009EO300001.

${ }^{2}$ Fisk, L.A., and Zhao, L., "The heliospheric magnetic field and the solar wind during the solar cycle," Proceedings of the International Astronomical Union, 4, 109-120, 2008, doi: 10.1017/S1743921309029160.

${ }^{3}$ de Jager, C., and Duhau, S., "Forecasting the parameters of sunspot cycle 24 and beyond," Journal of Atmospheric and Solar-Terrestrial Physics, 71(2), 239-245, 2009, doi: 10.1016/j.jastp.2008.11.006.

${ }^{4}$ Atwell, W., Tylka, A.J., Dietrich, W., Rojdev, K., and Matzking, C., "Sub-GLE Solar Particle Events and the Implications for Lightly-Shielded Systems Flown During an Era of Low Solar Activity,” Proceedings of 45 ${ }^{\text {th }}$ International Conference on Environmental Systems, Bellevue, WA, 2015.

${ }^{5}$ NOAA National Geophysical Data Center, “Space Weather,” URL: http://www.ngdc.noaa.gov/stp/spaceweather.html, accessed January 4, 2014.

${ }^{6}$ Space Weather Prediction Center, “Solar Proton Events Affecting the Earth Environment," URL: http://www.swpc.noaa.gov/ftpdir/indices/SPE.txt, accessed January 4, 2014.

${ }^{7}$ Badwhar, G.D., Cucinotta, F.A., and O’Neill, P.M., “An analysis of interplanetary space radiation exposure for various solar cycles,” Radiation Research, 138(2), 201-208, 1994.

${ }^{8}$ NOAA, “Solar Cycle Progression,” URL: http://www.swpc.noaa.gov/SolarCycle/index.html, accessed January 4, 2014.

${ }^{9}$ NOAA, "Predicted Sunspot Numbers and Radio Flux,” URL: http://www.swpc.noaa.gov/ftpdir/weekly/Predict.txt, accessed January 4, 2014.

${ }^{10}$ NOAA, "Solar Proton Events Affecting the Earth Environment: January 1976 - December 2013," URL: http://www.swpc.noaa.gov/ftpdir/indices/SPE.txt, accessed January 4, 2014. 
${ }^{11}$ Wilson, J.W., Badavi, F.F., Cucinotta, F.A., et al., "HZETRN: Description of a Free-Space Ion and Nucleon Transport and Shielding Computer Program,” NASA Technical Paper 3495, 1995. 